MIFP-09-18

\title{
Solutions to Hořava Gravity
}

\author{
H. Lü ${ }^{\dagger \star}$, Jianwei Mei ${ }^{\dagger}$ and C.N. Pope ${ }^{\dagger, \ddagger}$ \\ ${ }^{\dagger}$ George and Cynthia Woods Mitchell Institute for Fundamental Physics and Astronomy, \\ Texas A EM University, College Station, TX 77843-4242, USA \\ ${ }^{\star}$ Division of Applied Mathematics and Theoretical Physics, \\ China Institute for Advanced Study, \\ Central University of Finance and Economics, Beijing, 100081, China \\ $\ddagger D A M T P$, Centre for Mathematical Sciences, Cambridge University, \\ Wilberforce Road, Cambridge CB3 OWA, UK
}

\begin{abstract}
$\underline{\text { ABSTRACT }}$
Recently Hořava proposed a non-relativistic renormalisable theory of gravitation, which reduces to Einstein's general relativity at large distances, and that may provide a candidate for a UV completion of Einstein's theory. In this paper, we derive the full set of equations of motion, and then we obtain spherically symmetric solutions and discuss their properties. We also obtain solutions for the Friedman-Lemaître-Robertson-Walker cosmological metric.
\end{abstract}


Recently a new four-dimensional theory of gravity was proposed by Hořava [1], inspired by condensed matter models of dynamical critical systems. It has manifest threedimensional spatial general covariance and time-reparameterisation invariance, but only acquires four-dimensional general covariance in an infra-red large distance limit. It may be described in a language akin to the ADM 3+1 dimensional formulation of general relativity, but in which Einstein gravity is modified so that the full underlying four-dimensional covariance is broken. The nature of the modifications is governed by a rather strong principle of "detailed balance" [1].

In the ADM formalism, the four-dimensional metric of general relativity is parameterised as [2]

$$
d s_{4}^{2}=-N^{2} d t^{2}+g_{i j}\left(d x^{i}-N^{i} d t\right)\left(d x^{j}-N^{j} d t\right)
$$

where the lapse, shift and 3-metric $N, N^{i}$ and $g_{i j}$ are all functions of $t$ and $x^{i}$. In the simplest version of the theory in [1], the lapse function $N$ is viewed as a gauge field for time reparameterisations, and it is effectively restricted to depend only on $t$, but not the spatial coordinates $x^{i}$. A closer parallel with general relativity is achieved if this "projectability" restriction is relaxed. Thus one may take a broader view of the Hořava proposal as a class of theories in which the relative coefficients of the terms in the ADM decomposition of the Einstein-Hilbert action are modified, and additional terms involving higher spatial derivatives are included too. The higher derivative terms can improve the renormalisability of the theory, without the usual attendant problems of ghosts that would arise if higher time derivatives were present too. In this letter, we shall take the broader viewpoint (discussed also in [1]) in which the theory is essentially a class of $3+1$ dimensional modifications of general relativity.

The ADM decomposition (11) of the Einstein-Hilbert action is given by

$$
S_{E H}=\frac{1}{16 \pi G} \int d^{4} x \sqrt{g} N\left(K_{i j} K^{i j}-K^{2}+R-2 \Lambda\right),
$$

where $G$ is Newton's constant and $K_{i j}$ is defined by

$$
K_{i j}=\frac{1}{2 N}\left(\dot{g}_{i j}-\nabla_{i} N_{j}-\nabla_{j} N_{i}\right) .
$$

Here, a dot denotes a derivative with respect to $t$.

The action of the theory proposed by Hořava [1] can be written as

$$
\begin{aligned}
S & =\int d t d^{3} \mathbf{x}\left(\mathcal{L}_{0}+\mathcal{L}_{1}\right), \\
\mathcal{L}_{0} & =\sqrt{g} N\left\{\frac{2}{\kappa^{2}}\left(K_{i j} K^{i j}-\lambda K^{2}\right)+\frac{\kappa^{2} \mu^{2}\left(\Lambda_{W} R-3 \Lambda_{W}^{2}\right)}{8(1-3 \lambda)}\right\},
\end{aligned}
$$




$$
\mathcal{L}_{1}=\sqrt{g} N\left\{\frac{\kappa^{2} \mu^{2}(1-4 \lambda)}{32(1-3 \lambda)} R^{2}-\frac{\kappa^{2}}{2 w^{4}}\left(C_{i j}-\frac{\mu w^{2}}{2} R_{i j}\right)\left(C^{i j}-\frac{\mu w^{2}}{2} R^{i j}\right)\right\},
$$

where $\lambda, \kappa, \mu, w$ and $\Lambda_{W}$ are constant parameters, and $C_{i j}$ is the Cotton tensor, defined by

$$
C^{i j}=\epsilon^{i k \ell} \nabla_{k}\left(R_{\ell}^{j}-\frac{1}{4} R \delta_{\ell}^{j}\right)
$$

Comparing $\mathcal{L}_{0}$ to that of general relativity in the ADM formalism, the speed of light, Newton's constant and the cosmological constant emerge as

$$
c=\frac{\kappa^{2} \mu}{4} \sqrt{\frac{\Lambda_{W}}{1-3 \lambda}}, \quad G=\frac{\kappa^{2}}{32 \pi c}, \quad \Lambda=\frac{3}{2} \Lambda_{W} .
$$

(One can without loss of generality choose units so that $c=1$. Indeed this was done in (1) and (2).) Furthermore, the requirement that $\mathcal{L}_{0}$ be equivalent to the usual Einstein-Hilbert Lagrangian, and thus have four-dimensional general covariance, implies that one must take $\lambda=1$. In Hořava gravity, $\lambda$ represents a dynamical coupling constant, susceptible to quantum corrections [1]. In order to recover general relativity in the infra-red limit, the other dynamical coupling constants would need to flow so that $\mu \rightarrow 0, \Lambda_{W} \rightarrow \infty$ and $w \rightarrow \infty$, with $\mu^{2} \Lambda_{W}$ fixed. (There would also need to be a fine-tuning dynamical mechanism, presumably from a matter sector, to subtract the now-infinite cosmological constant proportional to $\mu^{2} \Lambda_{W}^{2}$.) Note from (6) that for $\lambda>\frac{1}{3}$, the cosmological constant $\Lambda_{W}$ has to be negative. The cosmological implications of the action (4) were recently discussed in [3, 4]. See also [5].

The constants $\mu$ and $w^{2}$ are real, and have their origin as the Newton constant and Chern-Simons coupling of Euclideanised three-dimensional topologically massive gravity [6]. However, if we make an analytic continuation of these parameters, namely $\mu \rightarrow \mathrm{i} \mu$, $w^{2} \rightarrow-\mathrm{i} w^{2}$, the four-dimensional action remains real, with the sign of all terms except $\left(K_{i j} K^{i j}-\lambda K^{2}\right)$ in (4) now being reversed. In this case, the emergent speed of light becomes $c=\frac{1}{4} \kappa^{2} \mu \sqrt{\Lambda_{W} /(3 \lambda-1)}$. The requirement that this speed be real implies that $\Lambda_{W}$ be positive for $\lambda>\frac{1}{3}$.

We now consider the equations of motion for the action (44). The equation following from the variation of $N$, is purely algebraic, and is given by

$$
\frac{2}{\kappa^{2}}\left(K_{i j} K^{i j}-\lambda K^{2}\right)-\frac{\kappa^{2} \mu^{2}\left(\Lambda_{W} R-3 \Lambda_{W}^{2}\right)}{8(1-3 \lambda)}-\frac{\kappa^{2} \mu^{2}(1-4 \lambda)}{32(1-3 \lambda)} R^{2}+\frac{\kappa^{2}}{2 w^{4}} Z_{i j} Z^{i j}=0,
$$

where

$$
Z_{i j} \equiv C_{i j}-\frac{\mu w^{2}}{2} R_{i j}
$$


The variation $\delta N^{i}$ implies

$$
\nabla_{k}\left(K^{k \ell}-\lambda K g^{k \ell}\right)=0
$$

The equations of motion due to the variation of $\delta g^{i j}$ are more complicated; they are given by

$$
\frac{2}{\kappa^{2}} E_{i j}^{(1)}-\frac{2 \lambda}{\kappa^{2}} E_{i j}^{(2)}+\frac{\kappa^{2} \mu^{2} \Lambda_{W}}{8(1-3 \lambda)} E_{i j}^{(3)}+\frac{\kappa^{2} \mu^{2}(1-4 \lambda)}{32(1-3 \lambda)} E_{i j}^{(4)}-\frac{\mu \kappa^{2}}{4 w^{2}} E_{i j}^{(5)}-\frac{\kappa^{2}}{2 w^{4}} E_{i j}^{(6)}=0,
$$

where

$$
\begin{aligned}
E_{i j}^{(1)}= & N_{i} \nabla_{k} K^{k}{ }_{j}+N_{j} \nabla_{k} K^{k}{ }_{i}-K^{k}{ }_{i} \nabla_{j} N_{k}-K^{k}{ }_{j} \nabla_{i} N_{k}-N^{k} \nabla_{k} K_{i j} \\
& -2 N K_{i k} K_{j}{ }^{k}-\frac{1}{2} N K^{k \ell} K_{k \ell} g_{i j}+N K K_{i j}+\dot{K}_{i j} \\
E_{i j}^{(2)}= & \frac{1}{2} N K^{2} g_{i j}+N_{i} \partial_{j} K+N_{j} \partial_{i} K-N^{k}\left(\partial_{k} K\right) g_{i j}+\dot{K} g_{i j} \\
E_{i j}^{(3)}= & N\left(R_{i j}-\frac{1}{2} R g_{i j}+\frac{3}{2} \Lambda_{W} g_{i j}\right)-\left(\nabla_{i} \nabla_{j}-g_{i j} \nabla_{k} \nabla^{k}\right) N \\
E_{i j}^{(4)}= & N R\left(2 R_{i j}-\frac{1}{2} R g_{i j}\right)-2\left(\nabla_{i} \nabla_{j}-g_{i j} \nabla_{k} \nabla^{k}\right)(N R) \\
E_{i j}^{(5)}= & \nabla_{k}\left[\nabla_{j}\left(N Z^{k}{ }_{i}\right)+\nabla_{i}\left(N Z^{k}{ }_{j}\right)\right]-\nabla_{k} \nabla^{k}\left(N Z_{i j}\right)-\nabla_{k} \nabla_{\ell}\left(N Z^{k \ell}\right) g_{i j}, \\
E_{i j}^{(6)}= & -\frac{1}{2} N Z_{k \ell} Z^{k \ell} g_{i j}+2 N Z_{i k} Z_{j}{ }^{k}-N\left(Z_{i k} C_{j}{ }^{k}+Z_{j k} C_{i}{ }^{k}\right)+N Z_{k \ell} C^{k \ell} g_{i j} \\
& -\frac{1}{2} \nabla_{k}\left[N \epsilon^{m k \ell}\left(Z_{m i} R_{j \ell}+Z_{m j} R_{i \ell}\right)\right]+\frac{1}{2} R_{\ell}^{n} \nabla_{n}\left[N \epsilon^{m k \ell}\left(Z_{m i} g_{k j}+Z_{m j} g_{k i}\right)\right] \\
& -\frac{1}{2} \nabla_{n}\left[N Z_{m}^{n} \epsilon^{m k \ell}\left(g_{k i} R_{j \ell}+g_{k j} R_{i \ell}\right)\right]-\frac{1}{2} \nabla_{n} \nabla^{n} \nabla_{k}\left[N \epsilon^{m k \ell}\left(Z_{m i} g_{j \ell}+Z_{m j} g_{i \ell}\right)\right] \\
& +\frac{1}{2} \nabla_{n}\left[\nabla_{i} \nabla_{k}\left(N Z_{m}{ }^{n} \epsilon^{m k \ell}\right) g_{j \ell}+\nabla_{j} \nabla_{k}\left(N Z_{m}^{n} \epsilon^{m k \ell}\right) g_{i \ell}\right] \\
& +\frac{1}{2} \nabla_{\ell}\left[\nabla_{i} \nabla_{k}\left(N Z_{m j} \epsilon^{m k \ell}\right)+\nabla_{j} \nabla_{k}\left(N Z_{m i} \epsilon^{m k \ell}\right)\right]-\nabla_{n} \nabla_{\ell} \nabla_{k}\left(N Z_{m}^{n} \epsilon^{m k \ell}\right) g_{i j} .
\end{aligned}
$$

(The equations of motion were also obtained in [4.) Note that in deriving these equations of motion, we have relaxed the "projectability" restriction and allowed the lapse function $N$ to depend on the spatial coordinates $x^{i}$ as well as $t$. Had we not done so, equation (77) would instead have to hold only when integrated over all space. Obtaining general relativity in an infra-red limit could then be problematical.

We may now seek static, spherically symmetric solutions with the metric ansatz

$$
d s^{2}=-N(r)^{2} d t^{2}+\frac{d r^{2}}{f(r)}+r^{2}\left(d \theta^{2}+\sin ^{2} \theta d \phi^{2}\right) .
$$

If we consider a system with the Lagrangian $\mathcal{L}_{0}$ only, we obtain the (A)dS Schwarzschild black hole with

$$
N^{2}=f=1-\frac{1}{2} \Lambda_{W} r^{2}-\frac{M}{r}
$$


The easiest way to obtain the solution for the full Lagrangian $\mathcal{L}_{0}+\mathcal{L}_{1}$ is to substitute the metric ansatz into the action, and then vary the functions $N$ and $f$. This is a valid procedure since the ansatz contains all the allowed singlets compatible with the $S O(3)$ action on the $S^{2}$. The resulting reduced Lagrangian, up to an overall scaling constant, is given by

$$
\mathcal{L}=\frac{N}{\sqrt{f}}\left(2-3 \Lambda_{W} r^{2}-2 f-2 r f^{\prime}+\frac{\lambda-1}{2 \Lambda_{W}} f^{\prime 2}-\frac{2 \lambda(f-1)}{\Lambda_{W} r} f^{\prime}+\frac{(2 \lambda-1)(f-1)^{2}}{\Lambda_{W} r^{2}}\right) .
$$

There are in total three solutions 1 The first solution is given by

$$
f=1+x^{2}, \quad x=\sqrt{-\Lambda_{W}} r .
$$

This is valid for all $\lambda$, but strangely enough, the function $N(r)$ is unconstrained. This suggests that if we have a hyperbolic spatial section, the Newtonian potential associated with $g_{t t}=-N^{2}$ can be an arbitrary function of $r$. (In fact, with $f$ given by (15)), we have verified that $N$ can be an arbitrary function of all the space-time coordinates.) As we shall show later, this particular feature is specific to the choice of coefficients that was made in [1] in order to satisfy the condition of "detailed balance."

There are two more solutions, in which both $f$ and $N$ are determined, given by

$$
f=1+x^{2}-\alpha x^{\frac{2 \lambda \pm \sqrt{6 \lambda-2}}{\lambda-1}}, \quad N=x^{-\frac{1+3 \lambda \pm 2 \sqrt{6 \lambda-2}}{\lambda-1}} \sqrt{f},
$$

where $\alpha$ is an integration constant. For the solution to be real, it is necessary that $\lambda>1 / 3$. It follows from (6) that $\Lambda_{W}$ is a negative cosmological constant if we consider the action (4); $\Lambda_{W}$ is a positive cosmological constant if we consider instead the action with the continuation $\mu \rightarrow \mathrm{i} \mu, w^{2} \rightarrow-\mathrm{i} w^{2}$. In the limit where $\lambda=1 / 3$, the function $f$ becomes that of the (A)dS black hole (13), but with twice the cosmological constant. The solution has a curvature singularity at $x=0$ for general $\lambda$. It also has a curvature singularity at $x=\infty$ if $(2 \pm \sqrt{6 \lambda-2})(\lambda-1)>0$.

It is of particular interest to investigate the $\lambda=1$ solution, in which case, the functions $f$ and $N$ are given by

$$
N^{2}=f=1+x^{2}-\alpha \sqrt{x} .
$$

The solution is asymptotically $\mathrm{AdS}_{4}$, with a horizon at $x=x_{+}$, where $x_{+}$is the largest root of $f$. The temperature is given by

$$
T=\frac{\left(3 x_{+}^{2}-1\right) \sqrt{-\Lambda_{W}}}{8 \pi x_{+}}
$$

\footnotetext{
${ }^{1}$ We have also verified that all the solutions indeed satisfy the full set of equations of motion (7), (9) and (10).
} 
There exists an extremal limit in which $\alpha=4 / 3^{3 / 4}$, with the horizon located at $x=1 / \sqrt{3}$, for which the temperature vanishes. The solution interpolates between the $\operatorname{AdS}_{2} \times S^{2}$ at the horizon and $\mathrm{AdS}_{4}$ at asymptotic infinity. The significant difference between the solution (17) and the usual AdS Schwarzschild black hole (13) suggests that general relativity is not always recovered at large distance 2

The action (44) was obtained by imposing the condition of detailed balance [1]. We may, however, entertain the idea of deviating slightly from detailed balance, by considering the Lagrangian

$$
\mathcal{L}=\mathcal{L}_{0}+\left(1-\epsilon^{2}\right) \mathcal{L}_{1}
$$

There exist two pure $\mathrm{AdS}_{4}$ solutions. The function $f$ is given by

$$
f=1-\frac{\Lambda_{W}}{1+\epsilon} r^{2}
$$

where $\epsilon$ can take both positive and negative values. The remaining equations imply that

$$
\epsilon\left(\Lambda_{W} r N+\left(1+\epsilon-\Lambda_{W} r^{2}\right) N^{\prime}\right)=0
$$

Thus we see that when detailed balance is satisfied, corresponding to $\epsilon=0$, the function $N$ is unconstrained, as previously noted. For $\epsilon \neq 0$, we find that $N^{2}=f$, giving rise to the $\mathrm{AdS}_{4}$ spacetimes.

The general solution for $f$ and $N$ can also be obtained, in the case of non-vanishing $\epsilon$. Owing to its complexity, we shall present only the special case where $\lambda=1$, for which it is given by

$$
N^{2}=f=1+\frac{x^{2}}{1-\epsilon^{2}}-\frac{\sqrt{\alpha^{2}\left(1-\epsilon^{2}\right) x+\epsilon^{2} x^{4}}}{1-\epsilon^{2}} .
$$

The large distance behaviour of the function $f$ is given by

$$
f=1+\frac{x^{2}}{1+\epsilon}-\frac{\alpha^{2}}{2 \epsilon x}+\mathcal{O}\left(x^{-4}\right)
$$

\footnotetext{
${ }^{2}$ It was observed in [7] that by writing the Schwarzschild metric in Painlevé-Gullstrand coordinates, it does in fact satisfy the projectability condition (with $N(t)=1$ ). We find that the AdS-Schwarzchild black hole can also be written in Painlevé-Gullstrand type coordinates, and it is given by$$
d s^{2}=-d t^{2}+\left(d r+\sqrt{\frac{M}{r}+\frac{\Lambda_{W}}{2} r^{2}} d t\right)^{2}+r^{2} d \Omega^{2} .
$$

Since in this coordinate system $g_{i j}$ is flat, it follows that the metric, with no modifications, gives an exact solution to the Hořava theory with $\lambda=1$, since the higher-order derivative corrections, from $\mathcal{L}_{1}$, vanish. However, owing to the negative cosmological constant $\Lambda_{W}$, the solution has no asymptotic $r \rightarrow \infty$ region. (Recall that one no longer has the freedom of four-dimensional general covariance.)
} 
Thus we see that for non-vanishing $\epsilon$, the metric has finite mass, which becomes divergent for the detailed-balance value $\epsilon=0$, in which case the function $f$ becomes the one given in (17). When $\epsilon=1$, the solution becomes the AdS-Schwarzschild black hole (13).

We may also look for cosmological solutions of the Friedman-Lemaitre-Robertson-Walker form

$$
d s^{2}=-d t^{2}+a^{2}(t)\left[\frac{d r^{2}}{1-k r^{2}}+r^{2}\left(d \theta^{2}+\sin ^{2} \theta d \phi^{2}\right)\right],
$$

where $k=1,0,-1$ corresponding to a closed, flat or open universe respectively. Supposing that the matter contribution is equivalent to an ideal fluid, we find that

$$
\begin{aligned}
\left(\frac{\dot{a}}{a}\right)^{2} & =\frac{2}{3 \lambda-1}\left(\frac{\Lambda_{W}}{2}+\frac{8 \pi G_{N} \rho}{3}-\frac{k}{a^{2}}+\frac{k^{2}}{2 \Lambda_{W} a^{4}}\right), \\
\frac{\ddot{a}}{a} & =\frac{2}{3 \lambda-1}\left(\frac{\Lambda_{W}}{2}-\frac{4 \pi G_{N}}{3}(\rho+3 p)-\frac{k^{2}}{2 \Lambda_{W} a^{4}}\right) .
\end{aligned}
$$

It is interesting to note that for $k=0$, there is no contribution from the higher-order derivative terms in the action. For $k \neq 0$, these contributions becomes dominant for small $a$, but weak at large $a$, implying that the cosmological solutions of general relativity are recovered at large scales.

For vacuum solutions with $p=\rho=0$, we have

$$
\left(\frac{\dot{a}}{a}\right)^{2}=\frac{\Lambda_{W}}{3 \lambda-1}\left(1-\frac{k}{\Lambda_{W} a^{2}}\right)^{2} .
$$

It follows from (6) that for the action (44), the right-hand side of the equation is negative definite for $\lambda>\frac{1}{3}$. Thus in this case, solutions only exist when $k=-1$, in which case $a$ is a constant given by $a^{2}=-1 / \Lambda_{W}$.

We can instead consider the action which is obtained from the analytic continuation $\mu \rightarrow \mathrm{i} \mu, w^{2} \rightarrow-\mathrm{i} w^{2}$ we discussed earlier. The reality condition for the speed of light now implies that $\Lambda_{W}$ is positive for $\lambda>\frac{1}{3}$. The solution is given by

$$
a^{2}=\frac{k}{\Lambda_{W}}+\alpha e^{\frac{2 \sqrt{\Lambda_{W}}}{\sqrt{3 \lambda-1}} t} .
$$

It is of interest to note that for $k=1$, there is a minimum scaling factor $a_{\text {min }}=1 / \sqrt{\Lambda_{W}}$.

In this paper, we have studied the recently-proposed non-relativistic and renormalisable gravity theory introduced in [1]. We derived the full set of equations of motion, and then considered the static, spherically-symmetric solutions. We found that there exists a solution where the spatial section is a hyperbolic space and the metric component $g_{t t}=-N^{2}$ can be an arbitrary function of all the space-time coordinates. We demonstrated that this feature occurs because certain coefficients in [1] are chosen to satisfy a condition of detailed balance. 
The system also admits $\mathrm{AdS}_{2} \times S^{2}$ vacuum solutions. In addition, there exist black hole solutions that interpolate between $\mathrm{AdS}_{2} \times S^{2}$ at the horizon and $\mathrm{AdS}_{4}$ at asymptotic infinity. The asymptotic fall-off of the metric for the black hole solutions is much slower than that of the AdS-Schwarzschild black hole in general relativity, suggesting that Einstein's gravity does not always appear to be recovered at large distance. We also obtained cosmological vacuum solutions.

\section{Acknowledgement}

We are grateful to Dick Arnowitt and Juan Maldacena for useful discussions, and to George Mitchell and Sheridan Lorenz for hospitality at Cook's Branch Conservancy where this work was completed. C.N.P. is supported in part by DOE grant DE-FG03-95ER40917.

\section{References}

[1] P. Hořava, Quantum gravity at a Lifshitz point. arXiv:0901.3775 [hep-th]

[2] R.L. Arnowitt, S. Deser and C.W. Misner, The dynamics of general relativity, "Gravitation: an introduction to current research", Louis Witten ed. (Wilew 1962), chapter 7, pp 227-265, arXiv:gr-qc/0405109.

[3] G. Calcagni, Cosmology of the Lifshitz universe, arXiv:0904.0829 [hep-th].

[4] E. Kiritsis and G. Kofinas, Hořava-Lifshitz cosmology, arXiv:0904.1334 [hep-th].

[5] P. Hořava, Membranes at quantum criticality, JHEP 0903, 020 (2009), arXiv: 0812.4287 [hep-th]. P. Hořava, Spectral dimension of the universe in quantum gravity at a Lifshitz point, arXiv:0902.3657 [hep-th]. A. Volovich and C. Wen, Correlation functions in non-relativistic holography, arXiv: 0903.2455 [hep-th]. T. Takahashi and J. Soda, Chiral primordial gravitational waves from a Lifshitz point, arXiv:0904.0554 [hep-th]. J. Kluson, Branes at quantum criticality, arXiv:0904.1343 [hep-th].

[6] S. Deser, R. Jackiw and S. Templeton, Topologically massive gauge theories, Annals Phys. 140, 372 (1982) [Erratum-ibid. 185, 406.1988 APNYA,281,409 (1988 APNYA,281,409-449.2000)].

[7] T. Sotiriou, M. Visser and S. Weinfurtner, Phenomenologically viable Lorentz-violating quantum gravity, arXiv:0904.4464 [hep-th]. 